\title{
Using Value Images to Adjust Intensity in 3D Renderings and Photographs
}

Reynold J. Bailey and Cindy M. Grimm

\author{
Washington University in St. Louis*
}

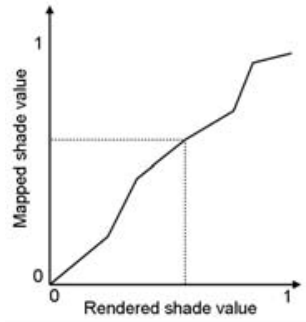

Figure 1: The value-map function.

\section{Introduction}

We present a technique for manipulating the intensity values in a $3 \mathrm{D}$ computer generated image or photograph. Artists often make value sketches of a scene before beginning the final painting. This value sketch is based on the lighting of the scene, but is not simply a faithful copy of it. The artist uses the value sketch to experiment with the placement and tone values of large masses of light and dark areas. This results in increased coherency and focus in the final image, and also guides the viewer to the important elements of the image. The artist can do this because our eyes are sensitive to local luminance variation [Livingstone 2002]. Photographers and computer animators, on the other hand, do not have the luxury of simply changing the tone values of a rendering to create the image they want. Instead, they manipulate the lighting and the surface qualities of the scene objects to achieve the desired effect. A typical rendered scene in a high-quality animation film will have anywhere from ten to a hundred lights, placed in strategic locations. Similarly, photographers and film studios use spotlights and other special-purpose lighting to create better photographs.

We present an alternative method for adjusting a 3D computer generated image or photograph to achieve the desired tonal values. The user supplies a value sketch (which can be very rough) of the scene. Our system finds a mapping from the current shade values to new shade values that preserves the relative lighting and keeps as much contrast as possible. This mapping can be applied to the entire scene in order to attain the desired global tone values or to individual regions to attain the desired local variation.

\section{Exposition}

The value sketch for a given $3 \mathrm{D}$ rendering or photograph can be created using a $2 \mathrm{D}$ paint program or by using traditional art media. The value-mapping function $V: \Re \rightarrow \Re$ maps the intensity value calculated by the lighting in the original scene to a new intensity value. The value-mapping function must be monotonically increasing, and bounded between 0 and 1 . These properties ensure that the relative intensity of the pixels in the original image is preserved. The default mapping is simply the identity function.

Given a desired value-sketch, the system finds the value-map function that minimizes the average difference between the value sketch

*email:rjb1/cmg@cse.wustl.edu and the rendered image and maintains contrast in the rendered image. To represent the value-map function we use a piece-wise linear function (see Figure 1). We could use a higher-order function, such as a quadratic spline function, but have found the linear function to be sufficient. Although the mapping is not smooth, if the divisions are small ( 0.1 or less) the effects are not noticeable ${ }^{1}$. We formulate the problem of solving for the value-map function as a constrained linear least-squares system.

In general, it is not possible to match the value image exactly. One approach is to divide the image into regions that have similar value tones, and find a map that is ideal for each region. We use a color image segmentation technique proposed by Dorin Comaniciu and Peter Meer [Comaniciu and Meer 1997] to do this. Figure 2 shows some typical results obtained using our technique.

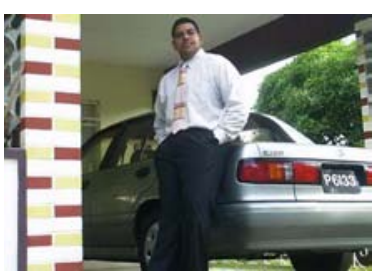

(a) Original image

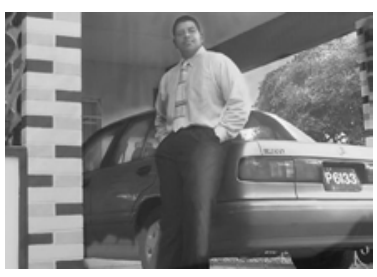

(c) Target intensity

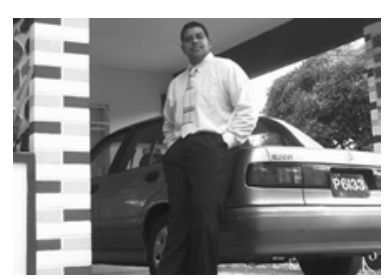

(b) Original intensity

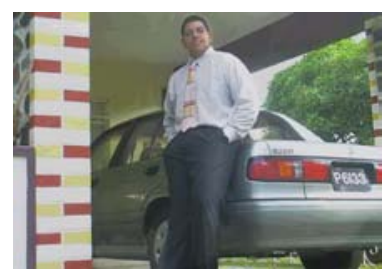

(d) Result

\section{Conclusion}

We have presented a technique that allows us to adjust the intensity of a rendered image to better match a user-defined value image without changing the overall lighting effect.

\section{References}

ComaniciU, D., And MeER, P., 1997. Robust analysis of feature spaces: Color image segmentation.

LiVingstone, M. 2002. Vision and Art: The Biology of Seeing. Harry N. Abrams, Inc.

\footnotetext{
${ }^{1}$ The discontinuities in the value map function result in $2^{\text {nd }}$ order discontinuities in the luminance function.
} 